\title{
Effect of Phosphorus fertilizer on growth and yield of cowpea (Vigna unguiculata)
}

\author{
*Nkaa, F.A. ${ }^{1}$, Nwokeocha ${ }^{2}$, O.W., Ihuoma, O. ${ }^{3}$ \\ ${ }^{1,2,3}$ Department of Plant Science and Biotechnology, Michael Okpara University of Agriculture, \\ Umudike, Abia state Nigeria.
}

\begin{abstract}
Phosphorus is important for cowpea production in many tropical African soils with inherent low phosphorus fertility. Most farmers in Africa, however, do not have access to phosphorus fertilizer. Selection of cowpea lines that produce good yield under low soil phosphorus or those with high phosphorus use efficiency can be a low input approach to solving this problem. This research work was conducted in pot trials at the teaching and research farm of Michael Okpara University of Agriculture, Umudike, in the south eastern part of Nigeria to determine the effect of different phosphorus levels on growth and yield of three varieties of cowpea (Vigna unguiculata (L.) Walp), obtained from the germplasm unit of International Institute of Tropical Agriculture, Ibadan. The experiment consists of five phosphorus levels $\left(0 \mathrm{kgha}^{-1}, 20 \mathrm{kgha}^{-1}, 40 \mathrm{kgha}^{-1}, 60 \mathrm{kgha}^{-1}\right.$ and $80 \mathrm{kgha}^{-1}$ ) each of which contains seven replicates. Phosphorus fertilizer significantly enhanced growth and yield characters of the cowpea varieties used; plant height, leaf area, number of leaves and number of branches in all the weeks of measurement were significantly improved. Phosphorus also had a significant effect ( $p>0.05)$ on seed yield per treatment, weight of 50 seeds, number of nodules, weight of nodules and total aboveground dry matter in all varieties used. However, variations were observed in the responses of the different cowpea varieties to phosphorus application. High yield values were observed in variety three; IT99K-573-2-1, followed by variety two; IT99K-573-1-1 and variety one; IT97K-499-35. Highest value in all the yield characters measured was observed in variety three: IT99K-573-2-1 at phosphorus fertilizer rate of 40kgha-1. When phosphorus is available, IT99K-573-2-1and 40kgha ${ }^{-1}$ phosphorus application rate is recommended.
\end{abstract}

Keywords: Phosphorus, Fertilizer, Cowpea, Growth, Yield, Umudike.

\section{Introduction}

Cowpea (Vigna unguiculata (L.) Walp) is an important grain legume in the dry savannah of the tropics covering 12 .5million hectares with annual production of about 3.million tons (FAO, 2005). Nigeria is one of the world's largest producer of cowpea with an average production of 2.92million tons followed by Niger with 1.10million tons (FAO, 2012).

Despite the dramatic increase in cowpea production in the sub-Saharan Africa, cowpea yields remain one of the lowest among all food legume crops, averaging at $450 \mathrm{~kg} / \mathrm{ha}$ in $2006-2008$, which is half of the estimated yields in all other developing regions. Its yields are very low due to several constraints including poor soil and use of low yield variety of seeds as planting material (Ecocrop, 2009).

Cowpea (Vigna unguiculata (L.) Walp) is a major staple food crop in sub-Saharan Africa, especially in the dry savannah regions of West Africa. The seeds are a major source of plant proteins and vitamins for man, feed for animals, and also a source of cash income (TJAI, 2010). The young leaves and immature pods are eaten as vegetables. There is a big market for the sale of cowpea grain and fodder in West Africa. In Nigeria, farmers who cut and store cowpea fodder for sale at the peak of the dry season have been found to increase their annual income by $25 \%$ (Dugje et al., 2009).

Cowpea also plays an important role in providing soil nitrogen to cereal crops (such as maize, millet, and sorghum) when grown in rotation, especially in areas where poor soil fertility is a problem. It does not require a high rate of nitrogen fertilization; its roots have nodules in which soil bacteria called Rhizobia inhabit and help to fix nitrogen from the air into the soil in the form of nitrates (Sheahan, 2012).

Cowpea can be grown under rain fed conditions as well as by using irrigation or residual moisture along river or lake flood plains during the dry season, provided that the range of minimum and maximum temperatures is between 28 and $30^{\circ} \mathrm{C}$ (night and day) during the growing season. Cowpea performs well in agro ecological zones where the rainfall range is between 500 and $1200 \mathrm{~mm} /$ year (Madamba et al., 2006). However, with the development of extra-early and early maturing cowpea varieties, the crop can thrive in the Sahel where the rainfall is less than $500 \mathrm{~mm} /$ year. It is tolerant of drought and well adapted to sandy and poor soils. It has little tolerance of salinity but is somewhat tolerant of soils high in aluminium. Like most legumes, it does not withstand waterlogged or flooded conditions.

However, best yields are obtained in well-drained sandy loam to clay loam soils with the $\mathrm{pH}$ between 6 and 7 (Ecocrop, 2009). 
Cowpea is a summer annual legume with trifoliate leaves. There are many cultivars, bred for diverse ecological niches, and they vary greatly in growth habit. Some are short, upright bush types, and others are tall and vine-like. Cowpea grows rapidly, reaching a height of 19-24 inches $(48-61 \mathrm{~cm})$ when grown under favourable conditions (Singh et al., 2003). Most root growth usually occurs within the topsoil layer, but in times of drought cowpea can grow a taproot as long as $8 \mathrm{ft}$ to reach moisture deeper in the soil profile (Madamba et al., 2006).

Cowpea does not require too much nitrogen fertilizer because it fixes its own nitrogen from the air using the nodules in its roots. However, in areas where soils are poor in nitrogen, there is a need to apply a small quantity of about $15 \mathrm{~kg}$ of nitrogen as a starter dose for a good crop. If too much nitrogen fertilizer is used, the plant will grow luxuriantly with poor grain yield. Cowpea requires more phosphorus than nitrogen in the form of single super phosphate or SUPA. About $30 \mathrm{~kg}$ of P/ha in the form of SUPA is recommended for cowpea production to help the crop to nodulate well and fix its own nitrogen from the air (FAO, 2005).

Tropical soils are inherently low in nutrients particularly nitrogen and phosphorus (Haruna et al., 2011). Phosphorus is among the most needed elements for crop production in many tropical soils.

Phosphorus is critical to cowpea yield because it is reported to stimulate growth, initiate nodule formation as well as influence the efficiency of the rhizobium-legume symbiosis (Haruna and Aliyu, 2011). It is required in large quantities in young cells such as shoot and root tips where metabolism is high and cell division is rapid. It also aids in flower initiation, seed and fruit development (Ndakemi and Dakora, 2007).

Phosphorus $(\mathrm{P})$ is among the most needed elements for crop production in many tropical soils. However, many tropical soils are P-deficient (Osodeke, 2005). The deficiency can be so acute in some soils of the savannah zone of western Africa that plant growth ceases as soon as the P stored in the seed is exhausted (Mokwunye and Bationo, 2002). Soil P-deficiencies primarily result from either inherent low levels of soil $\mathrm{P}$ or depletion of $\mathrm{P}$ through cultivation.

Legumes are phosphorus loving plants; they require phosphorus for growth and seed development and most especially in nitrogen fixation which is an energy-driving process. Legumes can fix up to $11-20 \mathrm{kgN} / \mathrm{ha}$ (Sanginga et al., 2000), but this is not achievable in the tropics because of low soil fertility and poor farming practices.

Application of phosphorus is therefore recommended for cowpea production on soils low in phosphorus. Careful application of phosphorus fertilizer to legumes is geared towards enhancing not only their growth and yield, but also nitrogen fixation. In Nigeria, legumes do not receive any form of mineral phosphorus fertilizer, they therefore entirely rely on the natural available soil phosphorus and other nutrients for nitrogenfixation and growth and this has resulted in lower yields (Singh et al., 2011).

One of the options of reducing low yields due to soil phosphorus content is to determine the best level of phosphorus fertilizer; as single super phosphate (SSP) so as to increase yield and returns from cowpea.

\section{Seed Collection}

\section{Materials And Methods}

Three certified cowpea varieties were obtained from the germplasm unit at International Institute of Tropical Agriculture (IITA), Ibadan Nigeria. The varieties are as follows: IT97K-499-35, IT99K-573-1-1, and IT99K-573-2-1. The genotypes were all semi-erect and early maturing varieties.

\section{Study Area}

The field experiment was conducted at the teaching and research farm of Michael Okpara University of Agriculture, Umudike. Umudike is in the tropical rainforest belt of Nigeria and lies on latitude $05^{\circ} 29^{\circ} \mathrm{N}$ and longitude $07^{\circ} 33^{\circ} \mathrm{E}$. Rainfall at Umudike has a bimodal distribution with one peak in July and the other in September and an average rainfall of $1275 \mathrm{~mm}$. It is $122 \mathrm{~m}$ above sea level and has a relative humidity of about $72 \%$. The monthly ambient temperature ranges from $17^{\circ} \mathrm{C}$ to $36^{\circ} \mathrm{C}$ (NRCRI meteorological report 2013).

The rainfall and air temperature data within the period of the experiment and the average rainfall and air temperature for ten years are shown in table 2.1.

Table 2.1 Meteorological Data for Umudike from January to July, 2013 and Average of Ten Years Data

\begin{tabular}{llllllll}
\hline & Jan & Feb & March & April & May & June & July \\
\hline $\begin{array}{l}\text { Rainfall }(\mathrm{mm}) \\
\text { Av. Rainfall }\end{array}$ & 3.1 & 107.1 & 68.5 & 259.0 & 436.3 & 240.1 & 359.3 \\
for 10yrs & 21 & 23.83 & 97.3 & 183.7 & 289.7 & 302.3 & 407.48 \\
$\begin{array}{l}\text { Max Temp. } \\
\left({ }^{\circ} \mathrm{C}\right)\end{array}$ & 32 & 34 & 33 & 32 & 32 & 30 & 30 \\
$\begin{array}{l}\text { Av. Max. } \\
\text { Temp. for }\end{array}$ & 32 & 34 & 33 & 33 & 32 & 31 & 30 \\
$\begin{array}{l}\text { 10yrs } \\
\text { Minimum }\end{array}$ & & & & & & & \\
\hline
\end{tabular}




\begin{tabular}{lrllllll}
\hline Temp. $\left({ }^{\circ} \mathrm{C}\right)$ & & & & 22 & 22 & 23 \\
Av. & Min. 19 & 22 & 23 & 24 & & \\
Temp. for & & & & & \\
10yrs
\end{tabular}

\section{Soil Collection}

The top soil $(0-15 \mathrm{~cm})$ was collected and $15 \mathrm{~g}$ was put into each nylon bag. The top soil was also collected randomly from the field and taken to the soil science laboratory in National Root Crop Research Institute for physiochemical analysis.

\section{Experimental Layout}

The experiment was a factorial combination of 3 cowpea lines and five $\mathrm{P}$ application rates in a randomised complete block design with seven replications.

Four seeds were sown in each bag on $11^{\text {th }}$ May 2013. Two weeks after planting, the seedlings were thinned to one plant per bag. A mixture of Karate ${ }^{\circledR} 2.5$ E.C. (50g lambda-cyhalothrin) per litre; $8 \mathrm{ml}$ in 1 litre of water) insecticide was sprayed twice to control insect pests during the experiment. The plants were grown to maturity and the crops harvested at physiological maturity when the pods had turned yellow.

\section{Treatment Application}

Phosphorus fertilizer as single super phosphate was applied using the ring method at five different levels; $0 \mathrm{~kg} / \mathrm{ha}\left(\mathrm{T}_{0}\right), 20 \mathrm{Kg} / \mathrm{ha}\left(\mathrm{T}_{1}\right), 40 \mathrm{Kg} / \mathrm{ha}\left(\mathrm{T}_{2}\right), 60 \mathrm{Kg} / \mathrm{ha}\left(\mathrm{T}_{3}\right)$, and $80 \mathrm{Kg} / \mathrm{ha}\left(\mathrm{T}_{4}\right)$ on $1^{\text {st }}$ June, 2013 (three weeks after planting). The right amount of $\mathrm{P}$ fertilizer was worked out for the different levels by taking into account the weight of soils in the bags $(15 \mathrm{Kg})$ and weight of 1 ha of top soil $\left(2.24 \times 10^{6} \mathrm{~kg}\right)$.

\section{Data Collection}

Data were collected on the vegetative and yield characteristics of the plants. The heights of three randomly selected crops were taken at two weeks interval starting from the first week after planting using metre rule. The leaves and branches of three randomly selected crops were counted on fortnight basis. The length and broadest width of three leaves randomly selected from three crops were measured using the metre rule, then the two parameters were multiplied with 2.325; a constant proposed by Osei-Yeboah et al., (1983) to obtain leaf area. Number of pods per plant was obtained by plucking all the pods from five randomly selected crops. The harvested pods were measured length and girth-wise using a thread and calibrating on a metre rule. Pod fresh weight per plant was obtained by weighing all the pods plucked from five randomly selected crops using Metler's weighing balance and dry weight per plant was obtained by drying all plucked pods from the five randomly selected crops in the oven at $50^{\circ} \mathrm{C}$ for 48 hours and weighing with Metler's weighing balance. Number of seeds per treatment was obtained by counting all the seeds gotten from the harvested pods in each treatment. Seed yield per treatment was obtained by weighing the seeds in all the pods harvested from each treatment and weight of 50 Seed obtained by weighing 50 randomly selected seeds from each treatment using Metler's weighing balance. Roots of five randomly selected crops were harvested and washed free of soil, the nodules were removed and counted and their weight determined using a Metler's weighing balance. To get the Total Above- Ground Dry Matter (TDM) of five randomly selected crops, these crops were harvested, washed in running tap, oven dried at $50^{\circ} \mathrm{C}$ for 48 hours and weighed. Roots of five randomly selected crops were harvested, washed in running tap, oven dried at $50^{\circ} \mathrm{C}$ for 48 hours and weighed using Metler's weighing balance to determine the root dry weight

\section{Statistical Analysis}

The data collected from different treatments were subjected to statistical analysis at $95 \%$ confidence interval using one-way and two-way ANOVA with SPSS version 17 and the means separated using the Duncan New multiple range tests.

\section{Result Of Soil Analyses}

\section{Results}

The top soil $(0-15 \mathrm{~cm})$ from the experimental field had the following properties: soil texture; sandy loam, $\mathrm{pH}-\mathrm{H}_{2} \mathrm{O} ; 4.8$, \%sand; 71.2, \%silt; 10.6, \%clay; 18.2, soil available phosphorus; $21.1 \mathrm{mg} / \mathrm{kg}$, \%nitrogen; $0.028, \%$ organic carbon; 0.45 , \%organic matter; 0.78 , exchangeable acids (Cmol/kg soil): $\mathrm{Ca} ; 3.2, \mathrm{Mg} ; 0.8, \mathrm{~K}$; $0.01, \mathrm{Na} ; 0.013, \mathrm{Al} ; 0.96$. 


\section{Growth Parameters \\ Plant Height}

The effect of phosphorus fertilizer on plant height is presented in Table 1. Generally, application of phosphorus fertilizer had significant effect on plant height in all the weeks after its application. Significant effect was also observed in the relationship between the varieties and phosphorus treatments.

Table 1:Effect Of Phosphorus Fertilizer On Plant Height

\begin{tabular}{lllllll}
\hline Varieties & Week 1 & Week 3 & Week 5 & Week7 & Week9 & Week 11 \\
\hline $\mathrm{V}_{1} \mathrm{~T}_{0}$ & $4.8 \pm 0.3^{\mathrm{ab}}$ & $9.8 \pm 0.6^{\mathrm{bc}}$ & $15.4 \pm 0.6^{\mathrm{a}}$ & $16.4 \pm 0.5^{\mathrm{a}}$ & $17.0 \pm 0.2^{\mathrm{bc}}$ & $19.5 \pm 0.6^{\mathrm{b}}$ \\
$\mathrm{V}_{1} \mathrm{~T}_{1}$ & $4.5 \pm 0.2^{\mathrm{b}}$ & $9.3 \pm 0.4^{\mathrm{c}}$ & $13.3 \pm 0.5^{\mathrm{c}}$ & $15.4 \pm 0.5^{\mathrm{b}}$ & $16.3 \pm 0.6^{\mathrm{c}}$ & $18.1 \pm 0.5^{\mathrm{b}}$ \\
$\mathrm{V}_{1} \mathrm{~T}_{2}$ & $4.8 \pm 0.2^{\mathrm{ab}}$ & $9.2 \pm 0.3^{\mathrm{c}}$ & $13.4 \pm 0.6^{\mathrm{c}}$ & $14.6 \pm 0.3^{\mathrm{c}}$ & $16.5 \pm 0.5^{\mathrm{c}}$ & $18.9 \pm 0.5^{\mathrm{b}}$ \\
$\mathrm{V}_{1} \mathrm{~T}_{3}$ & $5.2 \pm 0.2^{\mathrm{a}}$ & $10.5 \pm 0.2^{\mathrm{a}}$ & $16.0 \pm 0.3^{\mathrm{a}}$ & $16.6 \pm 0.3^{\mathrm{a}}$ & $18.1 \pm 0.5^{\mathrm{a}}$ & $20.7 \pm 0.3^{\mathrm{a}}$ \\
$\mathrm{V}_{1} \mathrm{~T}_{4}$ & $5.1 \pm 0.2^{\mathrm{a}}$ & $10.1 \pm 0.3^{\mathrm{ab}}$ & $14.3 \pm 0.5^{\mathrm{b}}$ & $16.0 \pm 0.5^{\mathrm{ab}}$ & $17.8 \pm 0.8^{\mathrm{ab}}$ & $19.5 \pm 0.6^{\mathrm{b}}$ \\
$\mathrm{V}_{2} \mathrm{~T}_{0}$ & $4.4 \pm 0.6^{\mathrm{b}}$ & $9.0 \pm 0.6^{\mathrm{b}}$ & $12.6 \pm 0.3^{\mathrm{ab}}$ & $13.8 \pm 0.6^{\mathrm{ab}}$ & $16.6 \pm 0.9^{\mathrm{a}}$ & $19.2 \pm 0.7^{\mathrm{a}}$ \\
$\mathrm{V}_{2} \mathrm{~T}_{1}$ & $4.8 \pm 0.4^{\mathrm{ab}}$ & $9.8 \pm 0.4^{\mathrm{b}}$ & $12.4 \pm 0.6^{\mathrm{bc}}$ & $13.1 \pm 0.2^{\mathrm{b}}$ & $14.2 \pm 0.5^{\mathrm{c}}$ & $17.8 \pm 0.3^{\mathrm{b}}$ \\
$\mathrm{V}_{2} \mathrm{~T}_{2}$ & $5.4 \pm 0.6^{\mathrm{a}}$ & $10.9 \pm 0.4^{\mathrm{a}}$ & $13.7 \pm 0.9^{\mathrm{a}}$ & $14.0 \pm 0.7^{\mathrm{a}}$ & $16.4 \pm 0.5^{\mathrm{a}}$ & $18.2 \pm 0.7^{\mathrm{ab}}$ \\
$\mathrm{V}_{2} \mathrm{~T}_{3}$ & $4.5 \pm 0.3^{\mathrm{ab}}$ & $9.2 \pm 0.3^{\mathrm{b}}$ & $11.4 \pm 0.5^{\mathrm{c}}$ & $13.8 \pm 0.2^{\mathrm{ab}}$ & $15.1 \pm 0.4^{\mathrm{bc}}$ & $18.5 \pm 0.5^{\mathrm{b}}$ \\
$\mathrm{V}_{2} \mathrm{~T}_{4}$ & $4.7 \pm 0.5^{\mathrm{ab}}$ & $9.5 \pm 0.7^{\mathrm{b}}$ & $13.3 \pm 0.6^{\mathrm{ab}}$ & $13.7 \pm 0.9^{\mathrm{ab}}$ & $16.2 \pm 0.6^{\mathrm{ab}}$ & $17.8 \pm 0.6^{\mathrm{b}}$ \\
$\mathrm{V}_{3} \mathrm{~T}_{0}$ & $4.7 \pm 0.5^{\mathrm{bc}}$ & $9.0 \pm 0.3^{\mathrm{b}}$ & $13.4 \pm 0.7^{\mathrm{c}}$ & $14.7 \pm 0.2^{\mathrm{b}}$ & $15.1 \pm 0.2^{\mathrm{b}}$ & $17.9 \pm 0.4^{\mathrm{b}}$ \\
$\mathrm{V}_{3} \mathrm{~T}_{1}$ & $4.5 \pm 0.3^{\mathrm{c}}$ & $9.1 \pm 0.3^{\mathrm{b}}$ & $13.3 \pm 0.6^{\mathrm{c}}$ & $14.7 \pm 0.2^{\mathrm{b}}$ & $15.7 \pm 0.5^{\mathrm{b}}$ & $17.8 \pm 0.7^{\mathrm{b}}$ \\
$\mathrm{V}_{3} \mathrm{~T}_{2}$ & $5.4 \pm 0.1^{\mathrm{a}}$ & $10.6 \pm 0.3^{\mathrm{a}}$ & $15.8 \pm 0.3^{\mathrm{a}}$ & $17.2 \pm 0.5^{\mathrm{a}}$ & $18.2 \pm 0.4^{\mathrm{a}}$ & $20.6 \pm 0.7^{\mathrm{a}}$ \\
$\mathrm{V}_{3} \mathrm{~T}_{3}$ & $4.7 \pm 0.3^{\mathrm{bc}}$ & $9.0 \pm 0.2^{\mathrm{b}}$ & $13.4 \pm 0.4^{\mathrm{c}}$ & $15.0 \pm 0.1^{\mathrm{b}}$ & $15.5 \pm 0.3^{\mathrm{b}}$ & $18.6 \pm 0.7^{\mathrm{b}}$ \\
$\mathrm{V}_{3} \mathrm{~T}_{4}$ & $5.1 \pm 0.3^{\mathrm{ab}}$ & $10.3 \pm 0.3^{\mathrm{a}}$ & $14.4 \pm 0.5^{\mathrm{b}}$ & $15.2 \pm 0.7^{\mathrm{b}}$ & $15.5 \pm 0.5^{\mathrm{b}}$ & $18.5 \pm 0.8^{\mathrm{b}}$ \\
\hline
\end{tabular}

* Means in the same column with the same superscript are not significantly different $(\mathrm{P} \geq 0.05) \pm$ Standard Deviation.

\section{Leaf Area}

The effect of phosphorus fertilizer on leaf area between varieties and between treatments is shown in Table 2 respectively. Phosphorus had significant effect on leaf area, and cowpea varieties showed significant variations in responses of their leaf area to phosphorus application. Interaction between the varieties and treatments was also significant.

Table 2:Effect Of Phosphorus Fertilizer On Leaf Area

\begin{tabular}{|c|c|c|c|c|c|c|}
\hline Varieties & Week 1 & Week 3 & Week 5 & Week7 & Week9 & Week 11 \\
\hline $\mathrm{V}_{1} \mathrm{~T}_{0}$ & $16.5 \pm 1.3^{\mathrm{d}}$ & $37.9 \pm 2.6^{\mathrm{d}}$ & $50.2 \pm 3.4^{c}$ & $64.3 \pm 3.4^{\mathrm{c}}$ & $84.4 \pm 10.4^{b}$ & $99.4 \pm 5.6^{b}$ \\
\hline $\mathrm{V}_{1} \mathrm{~T}_{1}$ & $23.3 \pm 0.9^{c}$ & $42.6 \pm 1.5^{\mathrm{cd}}$ & $71.7 \pm 1.2^{\mathrm{b}}$ & $85.5 \pm 6.1^{b}$ & $89.2 \pm 8.2^{\mathrm{ab}}$ & $152.2 \pm 30.5^{\mathrm{a}}$ \\
\hline $\mathrm{V}_{1} \mathrm{~T}_{2}$ & $32.4 \pm 5.3^{\mathrm{b}}$ & $58.7 \pm 3.2^{\mathrm{b}}$ & $101.3 \pm 2.2^{\mathrm{a}}$ & $91.4 \pm 15.1^{\mathrm{b}}$ & $96.9 \pm 12.2^{\mathrm{ab}}$ & $111.4 \pm 4.1^{b}$ \\
\hline $\mathrm{V}_{1} \mathrm{~T}_{3}$ & $42.2 \pm 4.5^{\mathrm{a}}$ & $64.4 \pm 4.1^{\mathrm{a}}$ & $95.8 \pm 4.5^{\mathrm{a}}$ & $113.3 \pm 1.3^{\mathrm{a}}$ & $110.2 \pm 18.7^{\mathrm{a}}$ & $125.7 \pm 12.2^{\mathrm{ab}}$ \\
\hline $\mathrm{V}_{1} \mathrm{~T}_{4}$ & $27.6 \pm 4.2^{\mathrm{bc}}$ & $45.2 \pm 3.2^{\mathrm{c}}$ & $69.4 \pm 3.8^{\mathrm{b}}$ & $90.3 \pm 1.5^{\mathrm{b}}$ & $94.9 \pm 10.8^{\mathrm{ab}}$ & $119.7 \pm 11.9^{b}$ \\
\hline $\mathrm{V}_{2} \mathrm{~T}_{0}$ & $30.1 \pm 7.5^{\mathrm{ab}}$ & $59.3 \pm 4.1^{\mathrm{b}}$ & $112.6 \pm 9.7^{\mathrm{a}}$ & $128.3 \pm 10.9^{b}$ & $134.7 \pm 8.8^{\mathrm{b}}$ & $180.9 \pm 19.3^{\mathrm{a}}$ \\
\hline $\mathrm{V}_{2} \mathrm{~T}_{1}$ & $31.1 \pm 3.7^{\mathrm{ab}}$ & $53.8 \pm 3.9^{\mathrm{b}}$ & $115.7 \pm 7.8^{\mathrm{a}}$ & $120.9 \pm 12.6^{\mathrm{b}}$ & $121.6 \pm 9.8^{\mathrm{b}}$ & $186.7 \pm 4.9^{\mathrm{a}}$ \\
\hline $\mathrm{V}_{2} \mathrm{~T}_{2}$ & $36.1 \pm 2.1^{\mathrm{a}}$ & $71.6 \pm 3.0^{\mathrm{a}}$ & $124.5 \pm 6.1^{\mathrm{a}}$ & $166.8 \pm 14.4^{\mathrm{a}}$ & $159.8 \pm 32.7^{\mathrm{ab}}$ & $166.1 \pm 20.7^{\mathrm{a}}$ \\
\hline $\mathrm{V}_{2} \mathrm{~T}_{3}$ & $22.7 \pm 2.1^{\mathrm{b}}$ & $41.5 \pm 6.9^{c}$ & $74.4 \pm 1.5^{\mathrm{b}}$ & $120.3 \pm 19.6^{\mathrm{b}}$ & $118.1 \pm 27.6^{b}$ & $191.1 \pm 17.3^{\mathrm{a}}$ \\
\hline $\mathrm{V}_{2} \mathrm{~T}_{4}$ & $25.3 \pm 5.7^{\mathrm{b}}$ & $62.1 \pm 6.1^{\mathrm{b}}$ & $121.5 \pm 10.9^{\mathrm{a}}$ & $136.2 \pm 7.9^{\mathrm{b}}$ & $193.4 \pm 23.3^{\mathrm{a}}$ & $190.0 \pm 23.8^{\mathrm{a}}$ \\
\hline $\mathrm{V}_{3} \mathrm{~T}_{0}$ & $20.2 \pm 2.1^{\mathrm{c}}$ & $37.9 \pm 0.8^{b}$ & $79.9 \pm 2.5^{\mathrm{bc}}$ & $104.5 \pm 5.7^{b}$ & $166.8 \pm 25.9^{\mathrm{a}}$ & $190.0 \pm 13.9^{\mathrm{a}}$ \\
\hline $\mathrm{V}_{3} \mathrm{~T}_{1}$ & $20.1 \pm 1.3^{c}$ & $37.5 \pm 0.8^{b}$ & $73.9 \pm 5.1^{\mathrm{c}}$ & $104.8 \pm 2.9^{b}$ & $105.1 \pm 8.1^{\mathrm{b}}$ & $116.9 \pm 15.5^{b}$ \\
\hline $\mathrm{V}_{3} \mathrm{~T}_{2}$ & $35.7 \pm 4.1^{\mathrm{a}}$ & $56.7 \pm 6.4^{\mathrm{a}}$ & $96.9 \pm 3.7^{\mathrm{a}}$ & $134.2 \pm 10.9^{\mathrm{a}}$ & $161.2 \pm 33.8^{\mathrm{a}}$ & $192.8 \pm 24.8^{\mathrm{a}}$ \\
\hline $\mathrm{V}_{3} \mathrm{~T}_{3}$ & $27.9 \pm 0.3^{\mathrm{b}}$ & $35.7 \pm 3.8^{\mathrm{b}}$ & $88.4 \pm 5.2^{\mathrm{ab}}$ & $133.7 \pm 11.7^{\mathrm{a}}$ & $147.0 \pm 14.9^{\mathrm{a}}$ & $165.6 \pm 29.1^{\mathrm{a}}$ \\
\hline $\mathrm{V}_{3} \mathrm{~T}_{4}$ & $23.5 \pm 3.5^{\mathrm{bc}}$ & $36.2 \pm 2.6^{\mathrm{b}}$ & $77.8 \pm 7.1^{\mathrm{c}}$ & $126.1 \pm 11.1^{\mathrm{a}}$ & $156.6 \pm 9.1^{\mathrm{a}}$ & $159.9 \pm 32.7^{\mathrm{ab}}$ \\
\hline
\end{tabular}

* Means in the same column with the same superscript are not significantly different $(\mathrm{P} \geq 0.05) \pm$ Standard Deviation.

\section{Number Of Leaves And Number Of Branches}

Phosphorus fertilizer application to the cowpea varieties had a positive effect on the number of leaves and number of branches produced. The result of the effect of phosphorus on number of leaves and number of branches between varieties and between treatments of cowpea is shown in Tables 3 and 4 respectively. There was a significant effect in number of leaves and number of branches by the cowpea varieties and phosphorus treatments as affected by phosphorus fertilizer application in all the weeks examined except in the $11^{\text {th }}$ week. The interaction between varieties of cowpea used and phosphorus treatment was also significant. 
Table 3:Effect Of Phosphorus Fertilizer On Number Of Leaves

\begin{tabular}{lllllll} 
Varieties & Week 1 & Week 3 & Week 5 & Week7 & Week & Week 11 \\
\hline $\mathrm{V}_{1} \mathrm{~T}_{0}$ & $3.7 \pm 1.2^{\mathrm{a}}$ & $6.0 \pm 1.7^{\mathrm{a}}$ & $15.0 \pm 1.7^{\mathrm{b}}$ & $23.3 \pm 3.5^{\mathrm{ab}}$ & $28.0 \pm 4.6^{\mathrm{b}}$ & $36.0 \pm 7.6^{\mathrm{b}}$ \\
$\mathrm{V}_{1} \mathrm{~T}_{1}$ & $3.0 \pm 0.0^{\mathrm{a}}$ & $5.0 \pm 0.0^{\mathrm{a}}$ & $16.0 \pm 1.7^{\mathrm{b}}$ & $24.0 \pm 1.7^{\mathrm{ab}}$ & $35.0 \pm 3.0^{\mathrm{ab}}$ & $45.7 \pm 5.7^{\mathrm{ab}}$ \\
$\mathrm{V}_{1} \mathrm{~T}_{2}$ & $3.0 \pm 0.0^{\mathrm{a}}$ & $6.0 \pm 1.7^{\mathrm{a}}$ & $18.0 \pm 3.5^{\mathrm{ab}}$ & $26.0 \pm 3.0^{\mathrm{ab}}$ & $33.0 \pm 3.5^{\text {ab }}$ & $41.3 \pm 4.9^{\mathrm{b}}$ \\
$\mathrm{V}_{1} \mathrm{~T}_{3}$ & $4.3 \pm 1.2^{\mathrm{a}}$ & $8.0 \pm 0.0^{\mathrm{a}}$ & $21.0 \pm 1.7^{\mathrm{a}}$ & $23.0 \pm 3.0^{\mathrm{b}}$ & $39.0 \pm 4.6^{\mathrm{a}}$ & $54.7 \pm 3.8^{\mathrm{a}}$ \\
$\mathrm{V}_{1} \mathrm{~T}_{4}$ & $3.7 \pm 1.2^{\mathrm{a}}$ & $8.0 \pm 3.0^{\mathrm{a}}$ & $21.0 \pm 1.7^{\mathrm{a}}$ & $29.0 \pm 3.0^{\mathrm{a}}$ & $37.0 \pm 6.2^{\mathrm{a}}$ & $45.0 \pm 4.6^{\mathrm{ab}}$ \\
$\mathrm{V}_{2} \mathrm{~T}_{0}$ & $3.0 \pm 0.0^{\mathrm{a}}$ & $8.0 \pm 0.0^{\mathrm{a}}$ & $21.0 \pm 1.7^{\mathrm{b}}$ & $39.0 \pm 3.5^{\mathrm{a}}$ & $43.0 \pm 4.6^{\mathrm{a}}$ & $55.7 \pm 3.5^{\mathrm{a}}$ \\
$\mathrm{V}_{2} \mathrm{~T}_{1}$ & $3.7 \pm 1.2^{\mathrm{a}}$ & $7.0 \pm 1.7^{\mathrm{a}}$ & $20.0 \pm 0.0^{\mathrm{b}}$ & $29.0 \pm 3.0^{\mathrm{b}}$ & $35.0 \pm 3.0^{\mathrm{b}}$ & $49.3 \pm 4.7^{\mathrm{a}}$ \\
$\mathrm{V}_{2} \mathrm{~T}_{2}$ & $4.3 \pm 1.2^{\mathrm{a}}$ & $9.0 \pm 1.7^{\mathrm{a}}$ & $24.0 \pm 1.7^{\mathrm{a}}$ & $28.0 \pm 6.2^{\mathrm{b}}$ & $35.0 \pm 6.0^{\mathrm{b}}$ & $48.0 \pm 10.5^{\mathrm{a}}$ \\
$\mathrm{V}_{2} \mathrm{~T}_{3}$ & $3.7 \pm 1.2^{\mathrm{a}}$ & $6.0 \pm 1.7^{\mathrm{a}}$ & $16.0 \pm 1.7^{\mathrm{c}}$ & $41.0 \pm 3.0^{\mathrm{a}}$ & $45.0 \pm 3.5^{\mathrm{a}}$ & $57.0 \pm 4.6^{\mathrm{a}}$ \\
$\mathrm{V}_{2} \mathrm{~T}_{4}$ & $3.0 \pm 0.0^{\mathrm{a}}$ & $7.0 \pm 1.7^{\mathrm{a}}$ & $22.0 \pm 1.7^{\mathrm{ab}}$ & $35.0 \pm 3.0^{\mathrm{a}}$ & $45.0 \pm 1.7^{\mathrm{a}}$ & $56.7 \pm 5.0^{\mathrm{a}}$ \\
$\mathrm{V}_{3} \mathrm{~T}_{0}$ & $3.0 \pm 0.0^{\mathrm{a}}$ & $6.7 \pm 1.2^{\mathrm{a}}$ & $22.0 \pm 1.7^{\mathrm{bc}}$ & $35.0 \pm 3.0^{\mathrm{b}}$ & $45.0 \pm 1.7^{\mathrm{b}}$ & $56.7 \pm 5.0^{\mathrm{a}}$ \\
$\mathrm{V}_{3} \mathrm{~T}_{1}$ & $3.7 \pm 1.2^{\mathrm{a}}$ & $7.3 \pm 1.2^{\mathrm{a}}$ & $21.0 \pm 1.7^{\mathrm{c}}$ & $39.0 \pm 1.7^{\mathrm{b}}$ & $57.0 \pm 4.6^{\mathrm{a}}$ & $61.3 \pm 5.9^{\mathrm{a}}$ \\
$\mathrm{V}_{3} \mathrm{~T}_{2}$ & $4.3 \pm 1.2^{\mathrm{a}}$ & $9.0 \pm 1.7^{\mathrm{a}}$ & $26.0 \pm 3.0^{\mathrm{a}}$ & $46.0 \pm 1.7^{\mathrm{a}}$ & $56.0 \pm 3.0^{\mathrm{a}}$ & $63.0 \pm 7.0^{\mathrm{a}}$ \\
$\mathrm{V}_{3} \mathrm{~T}_{3}$ & $3.0 \pm 0.0^{\mathrm{a}}$ & $7.3 \pm 1.2^{\mathrm{a}}$ & $25.0 \pm 1.7^{\mathrm{ab}}$ & $35.0 \pm 3.0^{\mathrm{b}}$ & $41.0 \pm 3.0^{\mathrm{b}}$ & $55.0 \pm 4.6^{\mathrm{a}}$ \\
$\mathrm{V}_{3} \mathrm{~T}_{4}$ & $3.7 \pm 1.2^{\mathrm{a}}$ & $8.0 \pm 0.0^{\mathrm{a}}$ & $24.0 \pm 1.7^{\mathrm{ab}}$ & $44.0 \pm 3.0^{\mathrm{a}}$ & $51.0 \pm 1.7^{\mathrm{a}}$ & $61.0 \pm 7.5^{\mathrm{a}}$ \\
\hline
\end{tabular}

* Means in the same column with the same superscript are not significantly different $(\mathrm{P} \geq 0.05) \pm$ Standard Deviation.

Table 4:Effect Of Phosphorus Fertilizer On Number Of Branches

\begin{tabular}{|c|c|c|c|c|c|c|}
\hline Varieties & Week 1 & Week 3 & Week 5 & Week7 & Week9 & Week 11 \\
\hline $\mathrm{V}_{1} \mathrm{~T}_{0}$ & $1.3 \pm 0.6^{\mathrm{a}}$ & $2.3 \pm 0.6^{\mathrm{a}}$ & $5.3 \pm 0.6^{b}$ & $7.0 \pm 1.0^{\mathrm{c}}$ & $9.7 \pm 1.5^{b}$ & $12.3 \pm 2.5^{b}$ \\
\hline $\mathrm{V}_{1} \mathrm{~T}_{1}$ & $1.0 \pm 0.0^{\mathrm{a}}$ & $2.0 \pm 0.0^{\mathrm{a}}$ & $5.7 \pm 0.6^{\mathrm{b}}$ & $8.3 \pm 0.6^{\mathrm{abc}}$ & $12.0 \pm 1.0^{\mathrm{ab}}$ & $15.3 \pm 1.5^{\mathrm{ab}}$ \\
\hline $\mathrm{V}_{1} \mathrm{~T}_{2}$ & $1.0 \pm 0.0^{\mathrm{a}}$ & $2.3 \pm 0.6^{\mathrm{a}}$ & $6.3 \pm 1.2^{\mathrm{ab}}$ & $9.0 \pm 1.0^{\mathrm{ab}}$ & $11.3 \pm 1.2^{\mathrm{ab}}$ & $14.3 \pm 1.5^{b}$ \\
\hline $\mathrm{V}_{1} \mathrm{~T}_{3}$ & $1.7 \pm 0.6^{\mathrm{a}}$ & $3.0 \pm 0.0^{\mathrm{a}}$ & $7.3 \pm 0.6^{\mathrm{a}}$ & $8.0 \pm 1.0^{\mathrm{bc}}$ & $13.3 \pm 1.5^{\mathrm{a}}$ & $18.7 \pm 1.5^{\mathrm{a}}$ \\
\hline $\mathrm{V}_{1} \mathrm{~T}_{4}$ & $1.3 \pm 0.6^{\mathrm{a}}$ & $3.0 \pm 1.0^{\mathrm{a}}$ & $7.3 \pm 0.6^{\mathrm{a}}$ & $10.0 \pm 1.0^{\mathrm{a}}$ & $12.7 \pm 2.1^{\mathrm{a}}$ & $15.3 \pm 1.5^{\mathrm{ab}}$ \\
\hline $\mathrm{V}_{2} \mathrm{~T}_{0}$ & $1.0 \pm 0.0^{\mathrm{a}}$ & $2.0 \pm 0.0^{\mathrm{b}}$ & $7.3 \pm 0.6^{b}$ & $13.3 \pm 1.2^{\mathrm{a}}$ & $14.7 \pm 1.5^{\mathrm{a}}$ & $18.3 \pm 1.5^{\mathrm{ab}}$ \\
\hline $\mathrm{V}_{2} \mathrm{~T}_{1}$ & $1.3 \pm 0.6^{\mathrm{a}}$ & $2.7 \pm 0.6^{\mathrm{ab}}$ & $7.0 \pm 0.0^{\mathrm{b}}$ & $10.0 \pm 0.0^{\mathrm{b}}$ & $12.0 \pm 1.0^{\mathrm{b}}$ & $15.3 \pm 1.5^{b}$ \\
\hline $\mathrm{V}_{2} \mathrm{~T}_{2}$ & $1.7 \pm 0.6^{\mathrm{a}}$ & $3.3 \pm 0.6^{\mathrm{a}}$ & $8.3 \pm 0.6^{\mathrm{a}}$ & $9.7 \pm 2.1^{\mathrm{b}}$ & $12.0 \pm 2.0^{\mathrm{b}}$ & $16.3 \pm 3.5^{\mathrm{ab}}$ \\
\hline $\mathrm{V}_{2} \mathrm{~T}_{3}$ & $1.3 \pm 0.6^{\mathrm{a}}$ & $2.3 \pm 0.6^{\mathrm{ab}}$ & $5.7 \pm 0.6^{\mathrm{c}}$ & $14.0 \pm 1.0^{\mathrm{a}}$ & $15.3 \pm 1.2^{\mathrm{a}}$ & $19.0 \pm 2.0^{\mathrm{ab}}$ \\
\hline $\mathrm{V}_{2} \mathrm{~T}_{4}$ & $1.0 \pm 0.0^{\mathrm{a}}$ & $2.7 \pm 0.6^{\mathrm{ab}}$ & $7.7 \pm 0.6^{\mathrm{ab}}$ & $13.7 \pm 0.6^{\mathrm{a}}$ & $16.7 \pm 0.6^{\mathrm{a}}$ & $20.3 \pm 1.5^{\mathrm{a}}$ \\
\hline $\mathrm{V}_{3} \mathrm{~T}_{0}$ & $1.0 \pm 0.0^{\mathrm{a}}$ & $2.3 \pm 0.6^{\mathrm{a}}$ & $7.7 \pm 0.6^{\mathrm{bc}}$ & $12.0 \pm 1.0^{\mathrm{b}}$ & $15.3 \pm 0.6^{\mathrm{b}}$ & $19.3 \pm 1.5^{\mathrm{a}}$ \\
\hline $\mathrm{V}_{3} \mathrm{~T}_{1}$ & $1.3 \pm 0.6^{\mathrm{a}}$ & $2.7 \pm 0.6^{\mathrm{a}}$ & $7.3 \pm 0.6^{\mathrm{c}}$ & $13.3 \pm 0.6^{\mathrm{b}}$ & $17.3 \pm 1.5^{\mathrm{a}}$ & $20.7 \pm 2.1^{\mathrm{a}}$ \\
\hline$V_{3} T_{2}$ & $1.7 \pm 0.6^{\mathrm{a}}$ & $3.3 \pm 0.6^{\mathrm{a}}$ & $9.0 \pm 1.0^{\mathrm{a}}$ & $15.7 \pm 0.6^{\mathrm{a}}$ & $19.0 \pm 1.0^{\mathrm{a}}$ & $22.0 \pm 2.0^{\mathrm{a}}$ \\
\hline $\mathrm{V}_{3} \mathrm{~T}_{3}$ & $1.0 \pm 0.0^{\mathrm{a}}$ & $2.7 \pm 0.6^{\mathrm{a}}$ & $8.7 \pm 0.6^{\mathrm{ab}}$ & $12.0 \pm 1.0^{\mathrm{b}}$ & $14.0 \pm 1.0^{\mathrm{b}}$ & $18.7 \pm 1.5^{\mathrm{a}}$ \\
\hline $\mathrm{V}_{3} \mathrm{~T}_{4}$ & $1.3 \pm 0.6^{\mathrm{a}}$ & $3.0 \pm 0.0^{\mathrm{a}}$ & $8.3 \pm 0.6^{\mathrm{abc}}$ & $15.0 \pm 1.0^{\mathrm{a}}$ & $18.0 \pm 1.0^{\mathrm{a}}$ & $21.3 \pm 2.5^{\mathrm{a}}$ \\
\hline
\end{tabular}

* Means in the same column with the same superscript are not significantly different $(\mathrm{P} \geq 0.05) \pm$ Standard Deviation.

\section{Yield Parameters Pod Yield}

The result of the effects of phosphorus fertilizer on pod fresh weight per plant, pod dry weight per plant, number of pods per plant, length of pod per plant and girth of pod per plant is presented on Table 5 .

Phosphorus fertilizer enhanced the fresh weight of pod per plant. There were variations among the cowpea varieties in responses of pod fresh weight per plant to phosphorus application but no significant difference was observed in all varieties.

Application of phosphorus fertilizer to the cowpea varieties had minimal effect on the dry weight of pods per plant and no significance difference was observed in relation to treatments of phosphorus applied.

The responses of number of pods per plant of the cowpea varieties to phosphorus application was not significant in varieties one (IT97K-499-35) and two (IT99K-573-1-1) but variety three (IT99K-573-2-1) showed variations in their response and thus the number of pod per plant of the variety was significantly enhanced by phosphorus application.

Phosphorus greatly increased the length of pod per plant and all the cowpea varieties used showed significant variations in response of pod length to phosphorus.

Application of phosphorus fertilizer had no effect on pod girth of the varieties used, and thus, interactions of cowpea varieties and phosphorus treatments on pod girth had no significance.

Table 5:Effect Of Phosphorus Fertilizer On Pod Yield

\begin{tabular}{llllll}
\hline Treatments & Fwpp $^{-1}$ & Dwpp $^{-1}$ & Nopp $^{-1}$ & Lopp $^{-1}$ & Gopp $^{-1}$ \\
\hline $\mathrm{V}_{1} \mathrm{~T}_{0}$ & $35.5 \pm 3.4^{\mathrm{a}}$ & $9.7 \pm 0.5^{\mathrm{a}}$ & $9.7 \pm 2.1^{\mathrm{a}}$ & $15.9 \pm 0.3^{\mathrm{a}}$ & $2.9 \pm 0.1^{\mathrm{a}}$ \\
$\mathrm{V}_{1} \mathrm{~T}_{1}$ & $42.9 \pm 12.4^{\mathrm{a}}$ & $11.0 \pm 1.7^{\mathrm{a}}$ & $10.3 \pm 2.5^{\mathrm{a}}$ & $12.6 \pm 0.7^{\mathrm{c}}$ & $3.0 \pm 0.2^{\mathrm{a}}$ \\
$\mathrm{V}_{1} \mathrm{~T}_{2}$ & $43.7 \pm 13.6^{\mathrm{a}}$ & $10.9 \pm 2.2^{\mathrm{a}}$ & $10.3 \pm 3.2^{\mathrm{a}}$ & $14.4 \pm 0.6^{\mathrm{b}}$ & $2.6 \pm 0.3^{\mathrm{a}}$ \\
$\mathrm{V}_{1} \mathrm{~T}_{3}$ & $60.9 \pm 3.7^{\mathrm{a}}$ & $11.9 \pm 2.2^{\mathrm{a}}$ & $16.7 \pm 4.7^{\mathrm{a}}$ & $16.7 \pm 0.9^{\mathrm{a}}$ & $3.0 \pm 0.5^{\mathrm{a}}$ \\
$\mathrm{V}_{1} \mathrm{~T}_{4}$ & $46.0 \pm 18.4^{\mathrm{a}}$ & $11.5 \pm 2.8^{\mathrm{a}}$ & $11.7 \pm 4.7^{\mathrm{a}}$ & $16.1 \pm 0.2^{\mathrm{a}}$ & $2.8 \pm 0.1^{\mathrm{a}}$ \\
\hline
\end{tabular}




\begin{tabular}{|c|c|c|c|c|c|}
\hline $\mathrm{V}_{2} \mathrm{~T}_{0}$ & $38.9 \pm 3.6^{b}$ & $10.1 \pm 0.3^{\mathrm{a}}$ & $10.3 \pm 1.5^{\mathrm{a}}$ & $12.6 \pm 1.1^{\mathrm{d}}$ & $3.3 \pm 0.2^{\mathrm{a}}$ \\
\hline $\mathrm{V}_{2} \mathrm{~T}_{1}$ & $38.7 \pm 3.6^{\mathrm{b}}$ & $10.4 \pm 0.7^{\mathrm{a}}$ & $9.3 \pm 1.5^{\mathrm{a}}$ & $14.8 \pm 0.5^{\mathrm{c}}$ & $3.0 \pm 0.3^{\mathrm{ab}}$ \\
\hline$V_{2} T_{2}$ & $50.5 \pm 4.1^{\mathrm{a}}$ & $10.9 \pm 1.0^{\mathrm{a}}$ & $11.7 \pm 0.6^{\mathrm{a}}$ & $20.2 \pm 0.8^{\mathrm{a}}$ & $3.2 \pm 0.2^{\mathrm{ab}}$ \\
\hline $\mathrm{V}_{2} \mathrm{~T}_{3}$ & $41.1 \pm 1.4^{\mathrm{ab}}$ & $9.8 \pm 0.4^{\mathrm{a}}$ & $10.0 \pm 1.0^{\mathrm{a}}$ & $19.5 \pm 0.5^{\mathrm{a}}$ & $2.9 \pm 0.1^{\mathrm{b}}$ \\
\hline $\mathrm{V}_{2} \mathrm{~T}_{4}$ & $45.4 \pm 9.5^{\mathrm{ab}}$ & $11.5 \pm 1.6^{\mathrm{a}}$ & $10.0 \pm 2.0^{\mathrm{a}}$ & $17.4 \pm 0.8^{\mathrm{b}}$ & $3.0 \pm 0.1^{\mathrm{ab}}$ \\
\hline $\mathrm{V}_{3} \mathrm{~T}_{0}$ & $55.1 \pm 4.1^{b}$ & $12.3 \pm 0.6^{\mathrm{ab}}$ & $12.0 \pm 1.0^{\mathrm{b}}$ & $20.4 \pm 0.8^{\mathrm{b}}$ & $3.2 \pm 0.2^{\mathrm{ab}}$ \\
\hline $\mathrm{V}_{3} \mathrm{~T}_{1}$ & $56.9 \pm 3.0^{\mathrm{b}}$ & $12.0 \pm 0.1^{\mathrm{b}}$ & $12.7 \pm 0.6^{b}$ & $20.7 \pm 0.3^{\mathrm{ab}}$ & $3.0 \pm 0.1^{\mathrm{b}}$ \\
\hline $\mathrm{V}_{3} \mathrm{~T}_{2}$ & $85.9 \pm 20.1^{\mathrm{a}}$ & $15.1 \pm 1.1^{\mathrm{a}}$ & $18.3 \pm 3.2^{\mathrm{a}}$ & $23.2 \pm 2.9^{\mathrm{a}}$ & $3.4 \pm 0.1^{\mathrm{a}}$ \\
\hline $\mathrm{V}_{3} \mathrm{~T}_{3}$ & $55.3 \pm 14.9^{\mathrm{b}}$ & $12.4 \pm 1.9^{\mathrm{ab}}$ & $12.7 \pm 2.5^{b}$ & $20.2 \pm 0.8^{\mathrm{b}}$ & $3.3 \pm 0.2^{\mathrm{ab}}$ \\
\hline$V_{3} T_{4}$ & $54.6 \pm 16.3^{b}$ & $12.2 \pm 2.7^{\mathrm{ab}}$ & $12.0 \pm 3.0^{\mathrm{b}}$ & $14.4 \pm 0.6^{\mathrm{c}}$ & $3.4 \pm 0.2^{\mathrm{a}}$ \\
\hline
\end{tabular}

* Means in the same column with the same superscript are not significantly different $(\mathrm{P} \geq 0.05) \pm$ Standard Deviation. $\mathbf{F W P P}^{-1}=$ Fresh weight of pod per plant. $\mathbf{D W P P}^{-1}=$ Dry weight of pod per plant. $\mathbf{N O P P}^{-1}=$ Number of pods per plant. $\mathbf{L O P P}^{-1}=$ Length of pod per plant. $\mathbf{G O P P}^{-1}=$ Girth of pod per plant

\section{Seed Yield}

The effect of phosphorus on number of seeds per treatment, seed yield per treatment and weight of 50 seeds per treatment is shown in Table 6. Generally, phosphorus fertilizer had positive effect on number of seed per treatment. Variability among the cowpea varieties in number of seed response to phosphorus application was more pronounced with variety three (IT99K-573-2-1) producing highest number of seeds. Interactions of varieties one and two (IT97K-499-35 and IT99K-573-1-1) and phosphorus treatments showed no significance while a remarkable significance was observed in variety three (IT99K-573-2-1). varieties.

Seed yield per treatment was significantly increased with phosphorus application in all three cowpea

Application of phosphorus fertilizer greatly enhanced the weight of 50 seeds in all cowpea varieties used. The interactions between cowpea varieties and phosphorus treatments on weight of 50 seeds were significant. Highest value in all the yield characters measured was observed in variety three: IT99K-573-2-1 at phosphorus fertilizer rate of $40 \mathrm{kgha}^{-1}$.

Table 6: Effect Of Phosphorus Fertilizer On Seed Yield

\begin{tabular}{llll}
\hline TREATMENTS & NST $^{-1}$ & SYT $^{-1}$ & W50S $^{\mathrm{a}}$ \\
\hline $\mathrm{V}_{1} \mathrm{~T}_{0}$ & $99.7 \pm 1.5^{\mathrm{a}}$ & $12.6 \pm 0.9^{\mathrm{b}}$ & $6.4 \pm 0.3^{\mathrm{c}}$ \\
$\mathrm{V}_{1} \mathrm{~T}_{1}$ & $97.7 \pm 27.1^{\mathrm{a}}$ & $12.8 \pm 4.9^{\mathrm{b}}$ & $6.6 \pm 0.6^{\mathrm{c}}$ \\
$\mathrm{V}_{1} \mathrm{~T}_{2}$ & $105.7 \pm 6.7^{\mathrm{a}}$ & $14.9 \pm 0.7^{\mathrm{b}}$ & $7.4 \pm 0.1^{\mathrm{b}}$ \\
$\mathrm{V}_{1} \mathrm{~T}_{3}$ & $149.3 \pm 23.1^{\mathrm{a}}$ & $23.5 \pm 3.1^{\mathrm{a}}$ & $8.2 \pm 0.1^{\mathrm{a}}$ \\
$\mathrm{V}_{1} \mathrm{~T}_{4}$ & $120.3 \pm 48.9^{\mathrm{a}}$ & $15.6 \pm 4.1^{\mathrm{b}}$ & $7.7^{\mathrm{a}} \pm 0.4^{\mathrm{ab}}$ \\
$\mathrm{V}_{2} \mathrm{~T}_{0}$ & $102.7 \pm 28.8^{\mathrm{a}}$ & $19.9 \pm 3.9^{\mathrm{a}}$ & $10.6 \pm 0.1^{\mathrm{a}}$ \\
$\mathrm{V}_{2} \mathrm{~T}_{1}$ & $96.0 \pm 26.9^{\mathrm{a}}$ & $19.1 \pm 3.9^{\mathrm{a}}$ & $10.5 \pm 0.2^{\mathrm{a}}$ \\
$\mathrm{V}_{2} \mathrm{~T}_{2}$ & $124.0 \pm 17.3^{\mathrm{a}}$ & $24.5 \pm 3.1^{\mathrm{a}}$ & $10.2 \pm 0.1^{\mathrm{b}}$ \\
$\mathrm{V}_{2} \mathrm{~T}_{3}$ & $95.7 \pm 8.7^{\mathrm{a}}$ & $19.2 \pm 1.3^{\mathrm{a}}$ & $9.6 \pm 0.1^{\mathrm{c}}$ \\
$\mathrm{V}_{2} \mathrm{~T}_{4}$ & $19.0 \pm 4.8^{\mathrm{a}}$ & $9.7 \pm 0.1^{\mathrm{c}}$ \\
$\mathrm{V}_{3} \mathrm{~T}_{0}$ & $100.0 \pm 25.9^{\mathrm{a}}$ & $20.9 \pm 2.4^{\mathrm{b}}$ & $9.7 \pm 0.2^{\mathrm{c}}$ \\
$\mathrm{V}_{3} \mathrm{~T}_{1}$ & $120.3 \pm 16.7^{\mathrm{b}}$ & $22.2 \pm 1.7^{\mathrm{b}}$ & $9.8 \pm 0.1^{\mathrm{c}}$ \\
$\mathrm{V}_{3} \mathrm{~T}_{2}$ & $119.3 \pm 13.6^{\mathrm{b}}$ & $36.6 \pm 5.6^{\mathrm{a}}$ & $10.9 \pm 0.1^{\mathrm{a}}$ \\
$\mathrm{V}_{3} \mathrm{~T}_{3}$ & $181.7 \pm 24.0^{\mathrm{a}}$ & $24.6 \pm 5.4^{\mathrm{b}}$ & $10.6 \pm 0.1^{\mathrm{b}}$ \\
$\mathrm{V}_{3} \mathrm{~T}_{4}$ & $125.7 \pm 26.6^{\mathrm{b}}$ & $21.5 \pm 5.5^{\mathrm{b}}$ & $10.7 \pm 0.2^{\mathrm{ab}}$ \\
\hline
\end{tabular}

*Means in the same column with the same superscript are not significantly different $(\mathrm{P} \geq 0.05) \pm$ Standard Deviation. $\mathbf{N S P}^{-1}=$ Number of seed per treatment. $\mathbf{S Y T}^{-1}=$ Seed yield per treatment. W50S $=$ Weight of 50 seeds

\section{Nodulation and Dry Matter Accumulation}

Both number and weight of nodules of cowpea varieties were significantly enhanced by the application of phosphorus fertilizer. The result of the effect of phosphorus on nodulation is presented in Table 7 and results of the effect of phosphorus on dry matter accumulation (root dry weight and the total above ground dry matter) are presented in Table 8.

Phosphorus had positive effects on total aboveground dry matter, the interactions between cowpea varieties and phosphorus treatments on total aboveground dry matter were significant. The cowpea varieties showed different responses to phosphorus treatment on root dry matter, the interaction was significant in varieties one and two (IT97K-499-35 and IT99K-573-1-1) but there was no significance in variety three (IT99K-573-2-1). Highest value for root dry weight was observed in variety two; IT99K-573-1-1 at phosphorus fertilizer rate of $60 \mathrm{kgha}^{-1}$ while that of total aboveground matter was observed in variety three; IT99K-573-2-1 at phosphorus fertilizer rate of $40 \mathrm{kgha}^{-1}$. 
Table 7:Effect of Phosphorus Fertilizer on Nodulation

\begin{tabular}{lll}
\hline Treatments & Nodule Number & Nodule Weight \\
\hline $\mathrm{V}_{1} \mathrm{~T}_{0}$ & $18.0 \pm 1.0^{\mathrm{a}}$ & $4.5 \pm 0.4^{\mathrm{c}}$ \\
$\mathrm{V}_{1} \mathrm{~T}_{1}$ & $14.3 \pm 0.6^{\mathrm{c}}$ & $5.2 \pm 0.2^{\mathrm{bc}}$ \\
$\mathrm{V}_{1} \mathrm{~T}_{2}$ & $15.3 \pm 0.6^{\mathrm{bc}}$ & $5.7 \pm 0.2^{\mathrm{b}}$ \\
$\mathrm{V}_{1} \mathrm{~T}_{3}$ & $17.3 \pm 0.6^{\mathrm{ab}}$ & $6.6 \pm 0.2^{\mathrm{a}}$ \\
$\mathrm{V}_{1} \mathrm{~T}_{4}$ & $16.0 \pm 2.0^{\mathrm{abc}}$ & $6.8 \pm 0.9^{\mathrm{a}}$ \\
$\mathrm{V}_{2} \mathrm{~T}_{0}$ & $25.0 \pm 3.0^{\mathrm{a}}$ & $9.8 \pm 0.4^{\mathrm{b}}$ \\
$\mathrm{V}_{2} \mathrm{~T}_{1}$ & $13.0 \pm 2.0^{\mathrm{b}}$ & $9.4 \pm 0.5^{\mathrm{b}}$ \\
$\mathrm{V}_{2} \mathrm{~T}_{2}$ & $23.0 \pm 1.0^{\mathrm{a}}$ & $11.2 \pm 0.4^{\mathrm{a}}$ \\
$\mathrm{V}_{2} \mathrm{~T}_{3}$ & $13.7 \pm 1.5^{\mathrm{b}}$ & $10.6 \pm 0.3^{\mathrm{a}}$ \\
$\mathrm{V}_{2} \mathrm{~T}_{4}$ & $24.0 \pm 4.0^{\mathrm{a}}$ & $11.3 \pm 0.5^{\mathrm{a}}$ \\
$\mathrm{V}_{3} \mathrm{~T}_{0}$ & $16.3 \pm 3.5^{\mathrm{b}}$ & $5.8 \pm 0.3^{\mathrm{c}}$ \\
$\mathrm{V}_{3} \mathrm{~T}_{1}$ & $14.0 \pm 3.0^{\mathrm{b}}$ & $6.6 \pm 0.4^{\mathrm{d}}$ \\
$\mathrm{V}_{3} \mathrm{~T}_{2}$ & $31.0 \pm 3.6^{\mathrm{a}}$ & $10.6 \pm 0.3^{\mathrm{c}}$ \\
$\mathrm{V}_{3} \mathrm{~T}_{3}$ & $18.7 \pm 3.1^{\mathrm{b}}$ & $11.2 \pm 0.3^{\mathrm{b}}$ \\
$\mathrm{V}_{3} \mathrm{~T}_{4}$ & $27.7 \pm 1.5^{\mathrm{a}}$ & $11.9 \pm 0.2^{\mathrm{a}}$ \\
\hline
\end{tabular}

* Means in the same column with the same superscript are not significantly different $(\mathrm{P} \geq 0.05) \pm$ Standard Deviation.

Table 8: Effect Of Phosphorus Fertilizer On Root Dry Weight And Total Aboveground Dry Matter

\begin{tabular}{lll}
\hline Treatments & Root Dry Weight & Total Aboveground Dry Matter \\
\hline $\mathrm{V}_{1} \mathrm{~T}_{0}$ & $0.9 \pm 0.1^{\mathrm{bc}}$ & $13.2 \pm 0.3^{\mathrm{c}}$ \\
$\mathrm{V}_{1} \mathrm{~T}_{1}$ & $0.8 \pm 0.1^{\mathrm{c}}$ & $14.3 \pm 0.3^{\mathrm{b}}$ \\
$\mathrm{V}_{1} \mathrm{~T}_{2}$ & $0.7 \pm 0.1^{\mathrm{c}}$ & $13.6 \pm 0.6^{\mathrm{bc}}$ \\
$\mathrm{V}_{1} \mathrm{~T}_{3}$ & $1.3 \pm 0.2^{\mathrm{a}}$ & $15.5 \pm 0.5^{\mathrm{a}}$ \\
$\mathrm{V}_{1} \mathrm{~T}_{4}$ & $1.1 \pm 0.2^{\mathrm{ab}}$ & $14.3 \pm 0.4^{\mathrm{b}}$ \\
$\mathrm{V}_{2} \mathrm{~T}_{0}$ & $0.8 \pm 0.6^{\mathrm{d}}$ & $14.0 \pm 0.4^{\mathrm{b}}$ \\
$\mathrm{V}_{2} \mathrm{~T}_{1}$ & $1.7 \pm 0.2^{\mathrm{bc}}$ & $22.2 \pm 0.3^{\mathrm{a}}$ \\
$\mathrm{V}_{2} \mathrm{~T}_{2}$ & $1.6 \pm 0.5^{\mathrm{c}}$ & $16.2 \pm 0,3^{\mathrm{b}}$ \\
$\mathrm{V}_{2} \mathrm{~T}_{3}$ & $2.3 \pm 0.2^{\mathrm{a}}$ & $13.4 \pm 3.3^{\mathrm{b}}$ \\
$\mathrm{V}_{2} \mathrm{~T}_{4}$ & $2.1 \pm 0.2^{\mathrm{ab}}$ & $15.6 \pm 0.2^{\mathrm{b}}$ \\
$\mathrm{V}_{3} \mathrm{~T}_{0}$ & $1.3 \pm 0.2^{\mathrm{a}}$ & $16.4 \pm 0.4^{\mathrm{b}}$ \\
$\mathrm{V}_{3} \mathrm{~T}_{1}$ & $1.1 \pm 0.2^{\mathrm{a}}$ & $16.3 \pm 1.4^{\mathrm{b}}$ \\
$\mathrm{V}_{3} \mathrm{~T}_{2}$ & $1.2 \pm 0.3^{\mathrm{a}}$ & $20.3 \pm 1.3^{\mathrm{a}}$ \\
$\mathrm{V}_{3} \mathrm{~T}_{3}$ & $1.0 \pm 0.3^{\mathrm{a}}$ & $17.5 \pm 0.5^{\mathrm{b}}$ \\
$\mathrm{V}_{3} \mathrm{~T}_{4}$ & $1.1 \pm 0.4^{\mathrm{a}}$ & $17.3 \pm 0.6^{\mathrm{b}}$ \\
\hline
\end{tabular}

* Means in the same column with the same superscript are not significantly different $(\mathrm{P} \geq 0.05) \pm$ Standard Deviation.

\section{Discussion}

Growth attributes such as plant height, leaf area, number of branches and number of leaves was significantly increased by the application of phosphorus fertilizer. This result is in conformity to the results observed by Krasilnikoff et al. (2003) and Nyoki et al. (2013). This could be attributed to the fact that phosphorus is required in large quantities in shoot and root tips where metabolism is high and cell division is rapid (Ndakidemi and Dakora, 2007). Thus, an indication that the cowpea varieties utilised the phosphorus fertilizer applied judiciously in growth and development processes.

Phosphorus application also improved some yield attributes taken into consideration in this study: pod fresh and dry weights, number of pods, length of pods, number of seeds, seed yield and weight of 50 seeds. These were found to be significantly different at 0.05 level of significance and this is in conformity with the findings of other workers (Okeleye and Okelana, 2000; Haruna and Usman, 2013; Odundo et al., 2001; Ntare and Bationo, 2002; Nyoki et al., 2013; Singh et al., 2011 and Ndor et al., 2012) who also discovered significant increase in yield of cowpea in response to phosphorus application. However, Agboola and Obigbesan (2001) reported that phosphorus application did not significantly increase cowpea yield but rather enhanced nodulation and phosphorus content of leaf and stem.

Highest value in all the yield characters measured was observed in variety three: IT99K-5732-1 at phosphorus fertilizer rate of 40 $\mathrm{kgha}^{-1}$, this contradicts the findings of Haruna and Usman (2013) who recorded highest yield at $30 \mathrm{~kg} \mathrm{ha}^{-1}$ in their experiment and Singh et al., (2011) who reported highest yield at $60 \mathrm{kgha}^{-1}$ and suggested that that may be the optimum as further application of phosphorus may or may not increase yield of cowpea. The significant response of the measured yield characters of cowpea to phosphorus application could be attributed to the role of phosphorus in seed formation and grain filling (Haruna, 2011). 
Phosphorus fertilizer application significantly enhanced nodule number and nodule weight in all the cowpea varieties used in this study. Highest value for nodule number was observed in variety three: IT99K-573-2-1 at phosphorus fertilizer rate of $40 \mathrm{kgha}^{-1}$ while that of nodule weight was observed in variety two: IT99K-573-1-1 at phosphorus fertilizer rate of $80 \mathrm{~kg} \mathrm{ha}^{-1}$. Significant increase in nodulation was also observed by Okeleye and Okelana (2000), Agboola and Obigbesan, (2001), Mokwunye and Bationo (2002), and Olaleye et al., (2011). These observations are quite true because phosphorus initiates nodule formation as well as influence the efficiency of the rhizobium-legume symbiosis thereby enhancing nitrogen fixation (Haruna and Aliyu, 2011).

The root dry weight and total above ground dry matter of the three varieties used showed remarkable significance in response to phosphorus application, the same result was observed by Okeleye and Okelana (2000) and Odundo et al., (2001).

\section{Conclusion}

The observed variations in the performance of the cowpea varieties used could provide a basis for selecting cowpea lines with greater agronomic efficiency in phosphorus deficient soil to reduce fertilizer cost. It could also be a pointer because pot experiment does not represent the real life situation and may not be a good methodology for evaluating varieties for release to farmers. However, it could be used in an initial assessment of large number of breeder lines. These variations could be important for selecting varieties suitable for a range of soil phosphorus conditions as well as to release to farmers on large scale production.

It could be concluded that IT99K-573-2-1 and phosphorus application rate of $40 \mathrm{kgha}^{-1}$ is ideal for soils low in phosphorus and is thus recommended for farmers in such regions for enhancement of cowpea yield.

\section{References}

[1]. Agboola, A. A. and Obigbesan, G. O., (2001). Effect of different sources and levels of P on the performance and P uptake of IfeBrown variety of cowpea. Ghana J. of Agric. Sci., 10 (1): 71-75.

[2]. Dugje, I. Y., Omoigai, L. O., Ekeleme, F., Karama, A. Y., and Ajeigbe, H., (2009). Farmer;s guide to cowpea production in west Africa. IITA, Ibadan, Nigeria. pp. 20.

[3]. Ecocrop, (2009). Ecocrop database, FAO. http:///ecocrop.fao.org/ecocrop/srv/en/home

[4]. FAO, (2005). Cowpea production database for Nigeria, 1990-2004. Food and Agricultural Organisation. http://www.faostat.fao.org/.

[5]. Food and Agriculture Organization (FAO)., 2012. Grassland species index. Vigna unguiculata http://www.fao.org/ag/AGP/AGPC/doc/Gbase/data/pf000090.htm

[6]. Haruna, I. M., (2011). Dry matter partititioning and grain yield potential in sesame (Sesamum indicum .L.) as influenced by poultry manure, nitrogen and phosphorus at Samaru, Nigeria. J. Agric.Technol., 7: 1571-1577.

[7]. Haruna, I. M., and Aliyu, L., (2011). Yield and economic returns of sesame (Sesamum indicum .L.) as influenced by poultry manure, nitrogen and phosphorus at Samaru, Nigeria. Elixir Agric., 39: 4884-4887.

[8]. Haruna, I. M., and Usman, A., (2013). Agronomic efficiency of cowpea varieties(Vigna unguiculata (L.) Walp) under varying phosphorus rates in Lafia, Nassarawa state, Nigeria. Asian J. Of Crop Sci., 5: 209-215.

[9]. Krasilnikoff, G., Gahoonia, T., and Erik-Nelson, N., (2003). Variation in phosphorus uptake by genotypes of cowpea (Vigna unguiculata (L.) Walp) due to differences in root and root hair length and induced rhizosphere processes. Plant and Soil., 251: 8391.

[10]. Madamba, R., Grubben, G. J. H., Asante, I. K., Akromah, R., (2006). Vigna unguiculata (L.) Walp record from protabase. Brink, M. and Belay, G. (Eds). PROT A (Plant Resources Of Tropical Africa), Wageningen, Netherlands.

[11]. Mokwunye, A. U. and Bationo, A., (2002). Meeting the phosphorus needs of soils and crops of west Africa: The role of indeginous phosphate rocks. In: Vanlauwe, B., Diels, J., Sanginga, N., and Merckx, R. (Eds). Integrated plant nutrition management in sub Saharan Africa; from concept to practise, CABI/IITA, Cormwell press, Trowbridge. pp.209-224.

[12]. Ndakidemi, P. A. and Dakora, F. D., (2007). Yield components of nodulated cowpea (Vigna unguiculata (L.) Walp) and maize (Zea mays) plants grown with exogenous phosphorus in different cropping systems. Aust. J. Exp. Agric. 47: 587-590.

[13]. Ndor E., Dauda, N., Abimuku, E., Azagatu, D., Anzaku, H., (2012). Effect of phosphorus fertilizer and spacing on growth, nodulation count and yield of cowpea(Vigna unguiculata (L.) Walp) in southern guinea savannah of Nigeria. Asian J. Agric. Sci., 4: 254-257.

[14]. Ntare, B. R. and Bationo, A., (2002). Effects of phosphorus on yield of cowpea cultivars intercropped with pearl millet on Psammentic Paleustalf in Niger. Spring. Link 32(2): 143-147.

[15]. Nyoki, D., Patrick, A., Ndakidemi, R., (2013). Economic benefits of Bradyrhizobium japonicum inoculation and phosphorus supplementation in cowpea(Vigna unguiculata (L.) Walp) grown in northern Tanzania. American J. of Research comm., 1(11): 173189 .

[16]. Okeleye, K. A. and Okelana, M. A O., (2000). Effect of phosphorus fertilizer on nodulation, growth, and yield of cowpea (Vigna unguiculata) varieties. Indian J. of Agric. Sci., 67(1): 10-12.

[17]. Olaleye, O., Fagbola, O., Abaidoo, R. C., and Ikeorah, N., (2011). Phosphorus response efficiency in cowpea genotypes. IITA, Ibadan. Ublished by Canadian Centre Of Sciences And Education. Pp.1-10.

[18]. Osei-Yeboah, Linday, S. J. I. and Gumbs, F. H., (1983). Estimating the leaf area of cowpea (Vigna unguiculata (L.) Walp) from linear measurement of terminal leaflet. Tropical Agric., 60: 144-150.

[19]. Osodeke, V. E., (2005). Determination of phosphorus requirements of cowpea (Vigna unguiculata (L.) Walp) in the acid soils of south eastern Nigeria using sorption isotherms. Global J. Of Agric.Sci., 4(2): 135-138.

[20]. Sanginga, N., Lyasse, O, and Singh, B. B., (2000). Phosphorus use efficiency and nitrogen balance of cowpea breeding lines in a low P soil of the derived savanna zone in West Africa. Plant and Soil 220: 119-128.

[21]. Sheahan, C.M., (2012). Plant guide for cowpea (Vigna unguiculata). USDA-Natural Resources Conservation Service, Cape May Plant Materials Center, Cape May, NJ. 
[22]. Singh, B., Ajeigbe, H. A., Tarawali, S. A., Ferdinez-Rivera, S., Abubakar, M., (2003). Improving the production and utilization of cowpea as food and fodder.Field Crops Resch., 84: 169-170.

[23]. Singh, A., Baoule, A. L., Ahmed, H. G., Aliyu, U., and Sokoto, M. B., (2011). Influence of phosphorus on the performance of cowpea (Vigna unguiculata (L.) Walp) varieties in the sudan savannah of Nigeria. Agric.Sci., 2: 313-317.

[24]. Thomas Jefferson Agricultural Institute (TJAI)., 2010. Cowpea: a versatile legume for hot, dry conditions. Columbia, MO. http://www.jeffersoninstitute.org/pubs/cowpea.shtml 